\title{
Calibration and Beam-Mapping Techniques for the Tianlai Pathfinder Dish Array
}

\author{
Trevor M. Oxholm \\ (Department of Physics, University of Wisconsin-Madison) \\ Madison, WI, USA
}

\begin{abstract}
Hydrogen intensity mapping is a promising technique to measuring large-scale structure. By detecting the integrated emission of the $21 \mathrm{~cm}$ HI signal from many galaxies simultaneously, astronomers can survey larger volumes of the universe than ever before. However, systematics related to foreground subtraction, variable antenna beam patterns, and calibration must be overcome before maps can be made of the $21 \mathrm{~cm}$ signal. The Tianlai Pathfinder array, a first-generation hydrogen intensity mapping experiment, is being constructed in order to practice and demonstrate our ability to overcome these challenges. We demonstrate two methods, delay-delay rate filtering and point source calibration, in order to understand the systematics of the Tianlai instrument. The methods used here will be used to help demonstrate the feasibility of full-scale hydrogen intensity mapping experiments, while contributing to the experimental toolset used for such experiments.
\end{abstract}

\section{Introduction}

The past two decades have exhibited a rapid growth in our understanding of the universe. Between the discovery of accelerating cosmic expansion (Riess, 1998), ever-increasing constraints on the properties of dark matter, and gravitational waves (LIGO Scientific, 2017), research in the field continues to yield exciting discoveries in fundamental physics and beyond. Cosmologists have developed the $\Lambda \mathrm{CDM}$ model, the leading candidate model of the universe, featuring a homogeneous, isotropic, flat background with nonrelativistic dark matter and a dark energy equation of state approximating a cosmological constant. The $\Lambda \mathrm{CDM}$ model has been repeatedly supported through observation and the cosmological parameters have been tightly constrained, but there are still gaps in astronomers' understanding of the cosmos. The mechanism behind dark energy, the nature of dark matter, and the verification of inflation remain burning questions hotly pursued by cosmologists.

Answering these questions relies on measurement of the structure of the universe on the largest scales. The earliest observations come from the cosmic microwave background (CMB), a remnant of the surface of last scattering. Missions such as WMAP (Hinshaw, 2013) and Planck (Adam, 2016) measure the statistics of the CMB to infer the large-scale structure of the universe and constrain the cosmological parameters. Large-scale structure can also be measured via optical surveys such as DES (Abbott, 2018) and LSST (LSST, 2012). These experiments survey the locations and redshifts of millions of galaxies, which are known to be present at the sites of dark matter haloes. By analyzing the statistics of these dark matter haloes, cosmologists can measure dark matter power spectrum, which is used to infer and test the cosmological parameters.

Though CMB measurements and galaxy surveys have been extremely successful in measuring the cosmological parameters, they both feature fundamental limitations. Notably, the CMB measures only a two-dimensional surface at redshift $z \sim 1100$, and galaxy surveys are difficult to extend past redshift $z \sim 2$. Ultimately, galaxy surveys cannot hope to extend beyond the epoch of galaxy formation and the first stars near redshift $z \sim 10$. Two other weaknesses of galaxy surveys are 
inaccurate redshift measurements and biased signals. Inaccurate redshifts weaken threedimensional statistics, as redshifts must be placed in large bins. Biases occur because optical surveys only observe galactic signals and remain ignorant of the underlying dark matter power spectrum except through lensing effects.

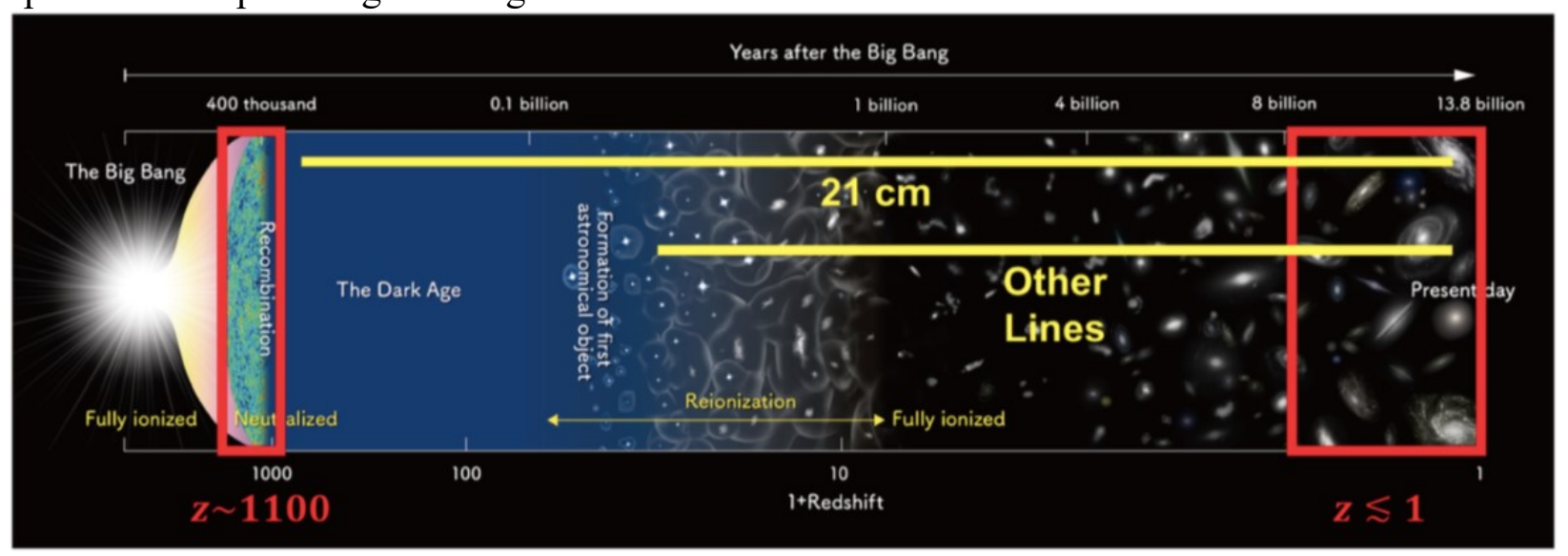

Figure 1 - Map of the universe between the surface of last scattering and present day. The red boxes represent the regions currently mapped by CMB and optical experiments, and the yellow lines show regions potentially mapped through line intensity mapping techniques. The image was taken from (Kovetz, 2017) ) .

Recent results suggest that the redshift range beyond the reach of galaxy surveys will be a rich testing ground for the measurement of cosmological parameters. Notably, the precise value for the Hubble Constant, $\mathrm{H}_{0}$, is under contention. $\mathrm{H}_{0}$ measurements using the $\mathrm{CMB}$ and supernovae differ by $\sim 20 \%$, suggesting that there may be systematic uncertainties in low-redshift measurements, or undiscovered science in the CMB era. Surveys beyond $z \sim 2$ are necessary to verify these results, which would profoundly shift astronomers' understanding of the universe and fundamental physics.

These physics, as well as structure formation, star and galaxy formation, and the epoch of reionization (EoR), lie in the history between the last scattering surface of the CMB and the recent universe. A new class of experiments known as line intensity mapping experiments are being developed with the hope of measuring the matter power spectrum in this region, overcoming the limitations of $\mathrm{CMB}$ experiments and optical surveys (Figure 1). Line intensity mapping involves the detection of a spectral line from the combined emission of many galaxies simultaneously, resulting in a map of the universe's matter distribution at precise redshifts. Whereas optical surveys rely on precise angular positions and relatively inaccurate spectroscopic measurements (redshift), line intensity mapping telescopes feature larger beams with higher frequency resolution. This approach promises a cost-effective way to obtain statistics on large volumes of the universe at high redshift.

There are many promising candidates for spectral lines to be used in line intensity mapping experiments. Hydrogen intensity mapping (HIM) involves measuring the redshifted $21-\mathrm{cm}$ line characteristic of the hyperfine structure of neutral hydrogen. Because of hydrogen's ubiquity in galaxies and the intergalactic medium throughout the history of the universe, HIM provides a tracer of an extensive portion of the universe, reaching from present times to the dark age. High-redshift HIM measurements would yield clues to the evolution of structure from the initial matter density 
perturbations, shedding light on the EoR, galaxy formation, and star formation. Low-redshift HIM measurements up to $z=2$ could yield clues of the nature of dark energy. Both high- and lowredshift experiments are currently in their first generation with the goal of establishing a proof of concept before the construction of more ambitious experiments.

Intensity mapping techniques have only recently been experimentally verified. The first discovery of 21-cm emission via intensity mapping occurred using the Green Bank Telescope (GBT) in 2010, by Chang et. al. (Chang, 2010). The experiment cross correlated the $21-\mathrm{cm}$ signal with the WiggleZ optical survey to measure the HI distribution in three dimensions between redshifts $z=0.53$ and $z=1.12$. HI has also tentatively been discovered at $z \sim 20$ by the EDGES experiment (Figure 2), and, if experimentally verified, will represent a groundbreaking discovery in the physics of the EoR (Bowman, 2018). CII has

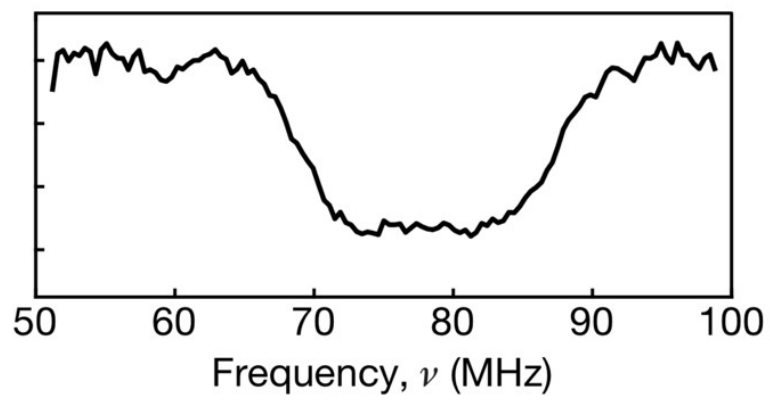

Figure 2 - The foreground-subtracted signal obtained by the EDGES experiment. The dip, if verified by other experiments, represents absorption in the 21-cm signal (Bowman, 2018) also tentatively been detected through a crosscorrelation between the Planck satellite and quasars from the Sloan Digital Sky Survey (Kovetz, 2017). CO intensity mapping has recently been detected with the Sunyaev-Zel'dovich Array (Keating, 2015).

All types of intensity mapping experiments face common challenges, including foreground removal, measuring the telescope response, and understanding the source signal itself. Emission lines are cloaked under galactic foregrounds featuring a smooth power law spectrum; in the case of $21-\mathrm{cm}$ line, the foreground signal is some 10,000 times larger than the hydrogen signal itself. Intensity mapping experiments must devise ways to extract the cosmological signal from beneath the foregrounds, and many candidate methods exist for this goal. However, many of these techniques rely on precise measurements of telescope beams, and accurate models of the foreground and the source signal.

Atmospheric foregrounds present another issue for future experiments. The ionosphere becomes opaque at frequencies below $\sim 10 \mathrm{MHz}$, rendering hydrogen intensity mapping above $\mathrm{z} \sim 100$ impossible with ground-based experiments. Therefore, a space-based mission will ultimately be necessary for measurements reaching into the dark ages, the era after recombination preceding the EoR. The SPHEREx experiment will use line intensity mapping of $\mathrm{Ha}$ and Lya to study the EoR, inflation, etc. The DARE experiment is a proposed satellite mission with the goal of measuring the EoR signal while under lunar orbit, thereby eliminating interference from the ionosphere and other earth-based sources of radio frequency interference. There are even discussions of a moonbased interferometer array, targeting $21-\mathrm{cm}$ emissions during the dark ages.

Here we discuss the Tianlai Pathfinder, a first-generation hydrogen intensity mapping experiment which uses methods of radio interferometry. The experiment was commissioned as a proofofconcept and testing ground for future hydrogen intensity mapping experiments. We use the 
Tianlai Pathfinder Dish Array to develop techniques to overcome the challenges characteristic of line intensity mapping experiments.

\section{Hydrogen intensity mapping with radio interferometry}

Most hydrogen intensity mapping experiments utilize radio interferometry on large arrays of telescopes. Radio interferometry is a powerful technique in which the signals from each telescope are correlated with the others (Figure 3 ). The resulting output for an array of $n$ telescope feeds is an $n(n+1) / 2$ dimensional array featuring $n(n-1) / 2$ cross-correlations and $n$ correlations. Thus, there is a great increase in the number of signals for a given measurement.

The key quantity used in radio interferometer arrays is the visibility $V_{\mathrm{v}}$, a complex quantity given by

$$
V_{\#}=\int A(s) I_{v}(s) e^{i \frac{2 \pi}{\lambda} \vec{b} \cdot s} d \Omega_{m} .
$$

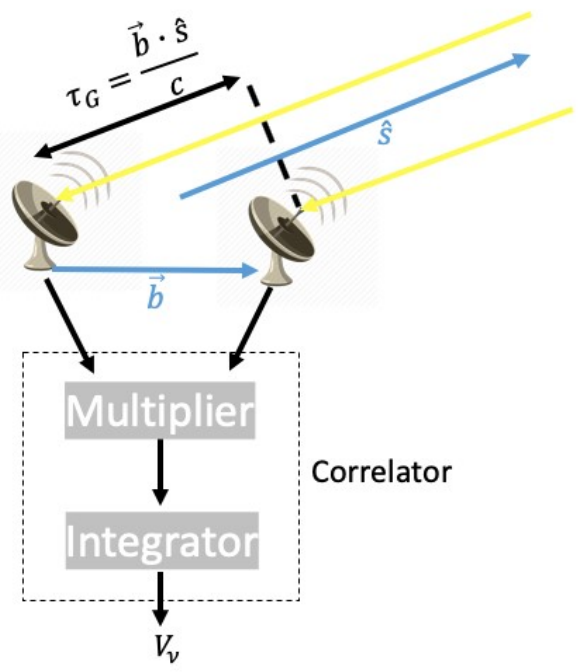

Here, $\hat{s}$ is the unit vector pointing to a source in the sky, $A(\hat{s})$ is the combined antenna response from the two correlated telescopes, $I_{\#}(\hat{s})$ is the intensity of the sky signal at a particular pointing and frequency, $b 2 \overrightarrow{\text { is }}$ the baseline vector Figure 3 - Diagram showing the correlation between two antennas, i.e. an element of an interferometer between the two telescopes, $\lambda$ is the incident photon wavelength, and $\Omega$ is the solid angle.

For line intensity mapping experiments, interferometer arrays are typically operated as driftscanning telescopes, maintaining a constant pointing with respect to Earth's surface. Thus, the product $b 2 \vec{s}$ is constantly changing as the sky rotates with respect to the antenna positions. The resulting visibility forms a complicated pattern, convolving the beam pattern with the positiondependent sky intensity. It is useful to consider the visibility pattern for a set of point sources (i.e. they

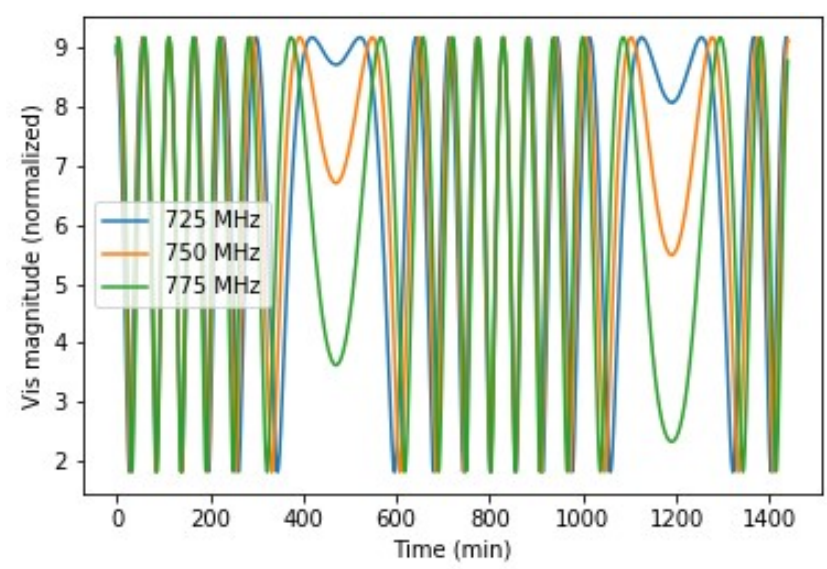
occupy a section of the sky smaller than the resolution of the telescopes)

$$
I_{v}(\hat{s})=\sum_{i} I_{v i} \delta\left(\hat{s}_{i}\right) .
$$

Figure 4 - Simulation performed by the author showing the 24 hour visibility signal for two sources near the North Celestial Pole

Here, the $i^{\text {th }}$ source has an intensity $I_{\#-\text { and }} \delta(\hat{s}-)$ is the Dirac delta function in its pointing direction. Thus, one obtains the visibility pattern 


$$
V_{v}=\sum_{i} I_{v i} A\left(\hat{s}_{i}\right) e^{i \frac{2 \pi}{\lambda} \vec{b} \cdot \hat{s}_{i}}
$$

Notably, the existence of the complex exponential term leads to interference effects between sources, resulting in a complicated oscillatory pattern in the output signal. Figure 4 shows a simulated visibility pattern over 24 hours (1440 minutes) for a pair of antennas pointing toward the North Pole, including only the two brightest point sources in the sky signal. We observe an offset sinusoidal output corresponding to the expected interference pattern. The features at $\sim 450$ minutes and $\sim 1170$ minutes are due to the brightest source.

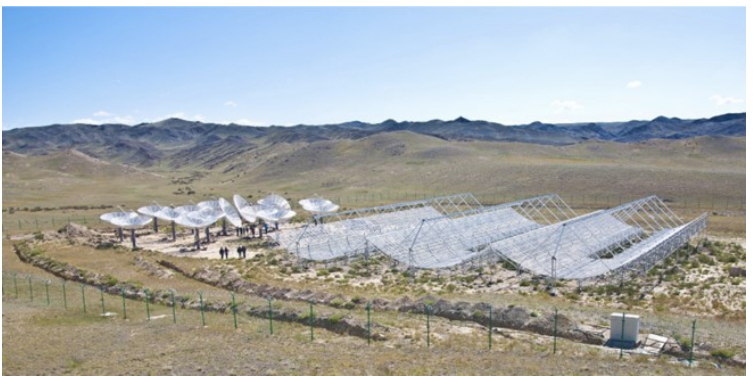

Figure 5 - The Tianlai dish and cylinder arrays. The image was taken from (Das, 2018)

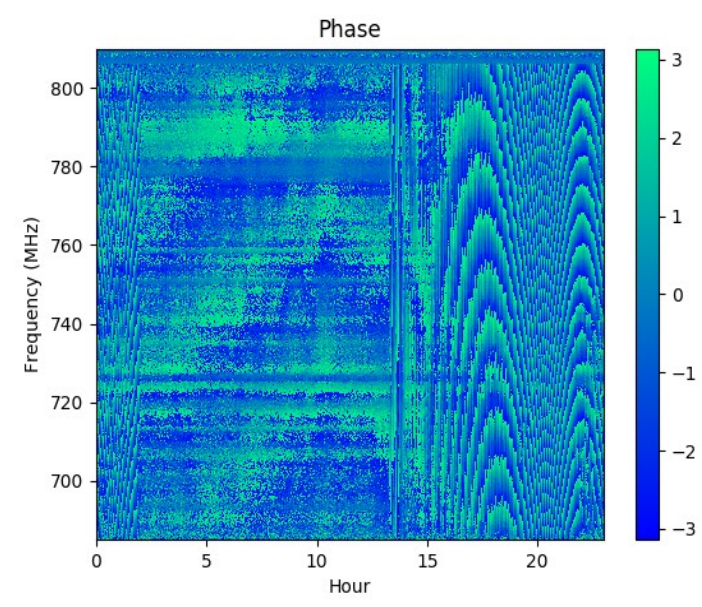

Figure 6 - Time-frequency map, produced by the author, over 24 hours for the Tianlai dish array pointing at the North Celestial Pole

\section{The Tianlai Dish Array}

The Tianlai Pathfinder Experiment, shown in Figure 5 features a dish array featuring sixteen $6 \mathrm{~m}$ dishes, and a cylinder array of three 15 $\mathrm{m} \times 40 \mathrm{~m}$ telescopes. All of the analysis generated for this project has related to the dish array. Each dish has two axes of rotation; one in latitude and one in right ascension. This allows the entire array to perform a drift scan at a particular declination, and if needed can alter its right ascension.

\section{Beam patterns}

The visibility signal in an interferometer array features a complicated convolution between the sky intensity pattern and beam intensity patterns. Complicated beam patterns present a challenge for radio interferometer arrays, as it is difficult to precisely understand the response of a single telescope due to incident radiation from all angles, let alone an entire array of telescopes. While the main lobe of a single antenna can be engineered accurately and precisely, the sidelobes at angles greater than $\sim 20-30$ degrees tend to yield complicated, frequency-dependent patterns. A single strong source in the far sidelobes can contribute to the visibility signal and is difficult to remove from

the global visibility signal. Figure 6 shows a typical time-frequency plot over 24 hours. Here, nighttime falls between hours $\sim 2$ and $\sim 13$, and the rest of the signal is dominated by the sun. Figure 7 shows the deviations between simulation and measurement for a single Tianlai dish beam, performed by our collaborators using a drone-based measurement. 
The use of delay-delay rate (DDR) filtering presents a possible means to removing point sources in the far sidelobes. A DDR transform involves taking the Fourier transform in both the time- and frequency-directions, resulting in delay and delay-rate spectra, respectively. Thus, a signal which is constant in time manifests itself as an impulse in delay space, and one that is constant in frequency corresponds to an impulse in delay-rate space.

A DDR filter can be used to isolate a single source, based on its fringe pattern in time and frequency space. The following algorithm was developed in (Parsons, 2009):

$$
V_{-?}(v, t)=\phi^{\mathrm{DE}}(v, t) \quad F_{\# \mathrm{G}} \mathrm{DE}^{\mathrm{DE}} F_{\mathrm{GH}}{ }^{\mathrm{DE}} G(\tau, f) F_{\mathrm{HG}} F_{\mathrm{L} \#} \phi(v, \tau) V_{-?}(v, t)
$$

Here, $V_{-?}(v, t)$ is the visibility cross-correlation between feeds $i$ and $j, \phi(v, \tau)$ is a phasor pointing toward the source to be filtered in phase space, $F$ and $F^{-1}$ are the Fourier Transform and Inverse Fourier Transforms mapping $v \rightarrow \tau$ (frequency to delay) and $t \rightarrow f$ (time to delay rate). $G(\tau, f)$ is a filter in delay-delay rate space, which can be taken as a high-pass filter in order to remove the specific source, or a low-pass filter to instead isolate the source.

\section{Point Source Calibration}

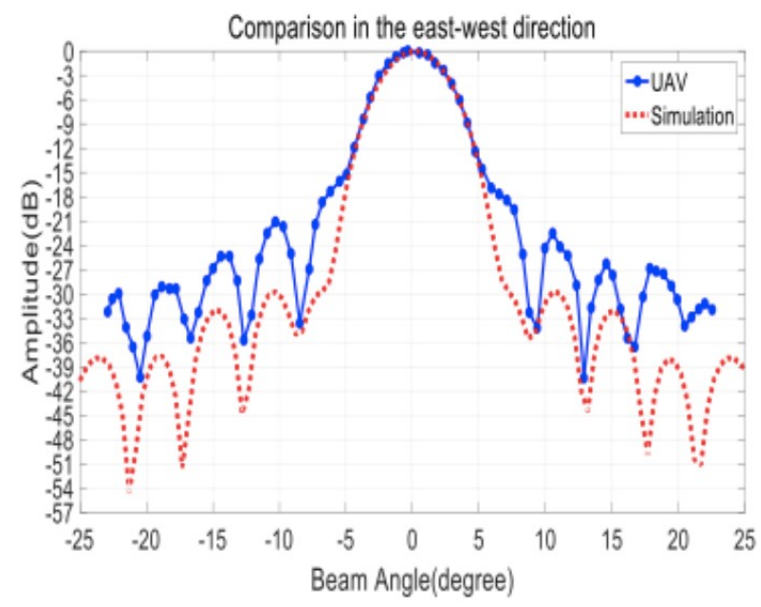

As with all telescopes, calibration presents an added challenge to the Tianlai dish experiment. Mainly low-noise amplifiers in the telescope electronics are temperature-dependent and are therefore susceptible to gain fluctuations. Thus, the output Figure 7 - Comparison between Tianlai dish beam patterns using CST simulations, vs. a drone-based measurement ( $\mathrm{Wu}$, to appear). The main lobe is in excellent agreement, whereas the sidelobes feature sizeable deviations. signal must be calibrated to an absolute amplitude and gain throughout the duration of a survey.

Strong point sources present a powerful means to calibration. Here, the visibility is dominated by a single source, yielding an output signal

$$
V_{v}=I_{v 0} A(\hat{s}) e^{i \frac{2 \pi}{\lambda} \vec{b} \cdot s}
$$

where $I_{\# N}$ is the output of the point source. This follows a much simpler form than the output due to many different sources, due to the absence of interference fringe patterns. Instead, the phase features a predictable time-dependence given by 


$$
\phi=\frac{2 \pi}{\lambda} \vec{b} \cdot \hat{s}=\frac{2 \pi}{\lambda}\left(b_{x} \cos \delta \sin \theta-b_{y}(\cos \delta \sin l \cos \theta-\sin \delta \cos l)\right)
$$

where $b_{x}$ and $b_{y}$ are the baseline components in the East-West and North-South directions, $\delta$ is the source declination, $l$ is the antenna latitude, and $\theta$ is the angle between the zenith of the beam and the right ascension of the source.

The slope of the phase is given by

$$
\frac{d \phi}{d t} \approx \frac{2 \pi}{\lambda}\left(\mathrm{b}_{\mathrm{x}} \cos \delta+b_{y} \cos \delta \sin l \theta\right) \omega_{e}
$$

where $\omega_{\mathrm{c}}$ is the angular speed of Earth's rotation.
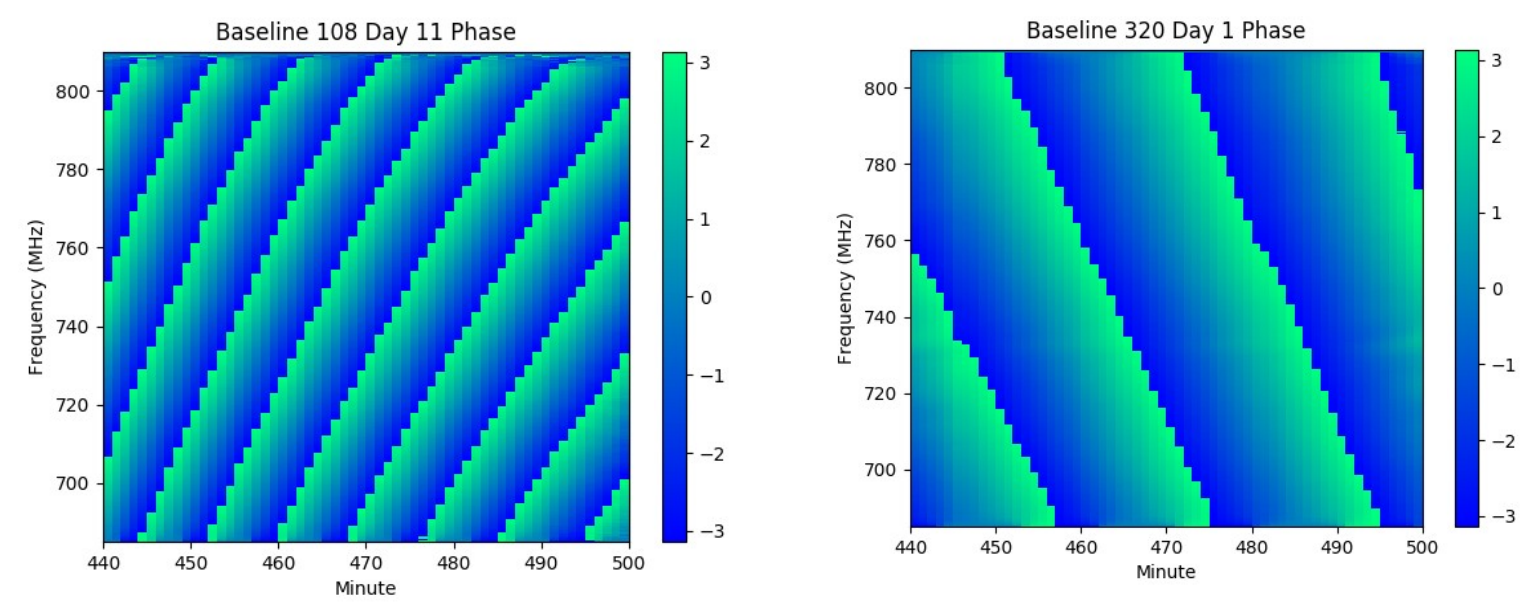

Figure 8 - Time-frequency maps over 60 minutes pointing at Cassiopeia $\mathrm{A}$, a strong radio point source. These are real data from the Tianlai instrument, analyzed by the author.

Time-vs-frequency plots of the antenna phase during a point source crossing, using Tianlai Dish Array data for two different baseline vectors, are shown Figure 8. The left figure corresponds to a $38 \mathrm{~m}$ baseline pointing Southwest (i.e. $b_{\mathrm{R}} \cong b_{\mathrm{Y}}$ ), whereas the right figure is an $8.8 \mathrm{~m}$ baseline pointing East $\left(b_{Y}=0\right)$. Notably, the longer baseline features more rapidly varying fringes than the shorter one. Additionally, the East-pointing baseline features a phase slope that is exactly linear in time, whereas the Southwest baseline phase slope deviates from linearity. These differences are all consistent with our expectations from the phase slope formula. We systematically analyze the phase slopes from these plots for many days and baselines, in order to understand properties of the telescope.

The main weaknesses of point source calibration are that (i) it can be only be performed once per day without changing the telescopes' East-West pointing, and (ii) the telescope must be pointing at a declination that corresponds to a specific source. Indeed, there are many strong radio point 
sources including Cassiopeia A, Cygnus A, Taurus A, etc., but many declinations including the North Pole have no such sources. Thus, the telescopes must be repointed for every calibration, which leads to additional systematics in the telescope electronics and physical machinery.

Sky calibration and noise source calibration are two potential means to overcoming the limitations of point source calibration. Sky calibration involves comparing each time and frequency pixel to its average value over many nights. Overall drifts in magnitude and phase can be calibrated out continuously. However, this technique relies on statistics over many nights and accurate accounting of systematics in the telescope system. Noise source calibration involves the use of a powerful emitter several kilometers from the telescope, which emits a characteristic pattern every few minutes and dwarfs the sky signal. The resulting signal can again be used to calibrate the magnitude and phase of the telescope signal. However, noise source calibration is geometrydependent, as the absorbed signal will change with antenna pointing. Noise source calibration also removes data from the sky signal. Both noise source calibration and sky calibration are currently being investigated to compliment point source calibration for Tianlai data.

\section{Results and Discussion}

Delay-delay rate filtering scheme shows promise as a means of subtracting parasitic sources appearing in the sidelobes of the instrument. The subfigures in Figure 9 shows time-frequency maps after various steps in the of delay-delay rate filtering of Cassiopeia A on a simulated sky. Here, the dishes are pointing at the North Celestial Pole, and the sky signal includes the two brightest North Pole sources in addition to Cassiopeia A.

The first row of subfigures in Figure 9 (i) shows the simulation results assuming a perfectly Gaussian beam pattern, i.e. the sidelobe pattern is uniform. The three subfigures show timefrequency maps of the signal (ia) unaltered, (ib) post-DDR filter, (ic) assuming Cassiopeia A was perfectly removed. Here, there are no visible deviations between figures (ib) and (ic), suggesting near-perfect filtering.

The second row of subfigures in Figure 9 (ii) displays results assuming a simulated beam, featuring azimuthal dependencies in the sidelobes but no frequency-dependence in the gain. Comparing the DDR-filtered signal (iib) to the perfectly filtered signal (iic), we see that the Cassiopeia A signal was not perfectly removed, as rapidly-varying fringes are still present. This is due to a complicated convolution between gain variations and fringes due to the astrophysical signal. These results demonstrate that while the DDR filter works well, it is no replacement for having precise knowledge of the beam pattern into the far sidelobes.

Point source calibration shows great promise in increasing our understanding of the instrument. The calibration was performed using Cassiopeia A, a known strong radio point source in the Northern hemisphere. Data were taken for 11 consecutive days, with the antennas constantly pointed at Cassiopeia A's declination. The peak magnitude and phases were both analyzed using the following scheme, developed by the author: 
i. For each frequency channel, obtain the peak magnitude and the phase slope during the Cassiopeia A crossing. For the phase slope calculation, a phase unwrapping algorithm is used.

ii. For the magnitude, find the mean and percent deviation compared to the average over 11 days, for each frequency channel individually. Do the same for the slope of a line fitted to the of the unwrapped phase.

iii. Produce a histogram of the percent deviations. There should be (number of frequency channels) $\mathrm{x}$ (number of days) entries.

ia
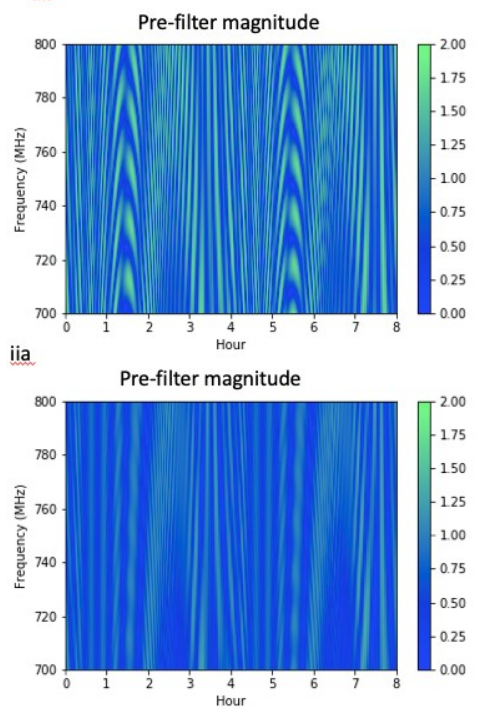

ib

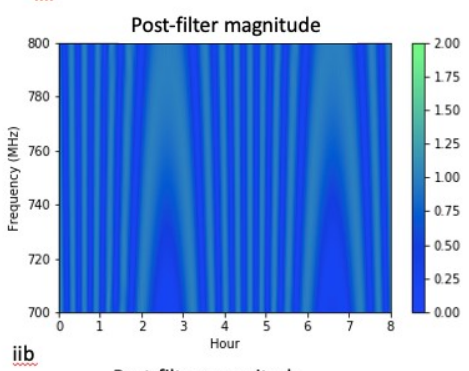

iib

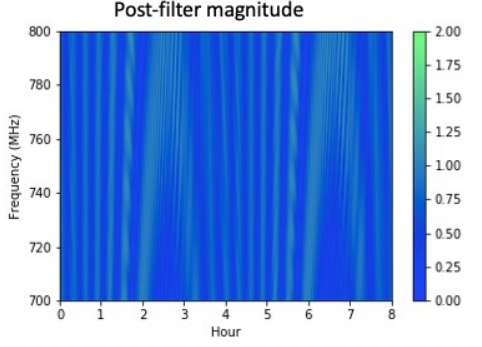

ic
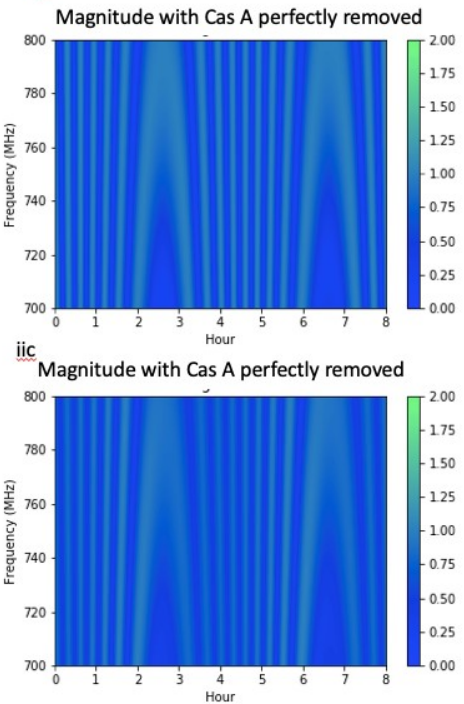

Figure 9 - Demonstration of DDR filtering of a single point source, using simulated sky patterns and analysis code developed by the author. ia, ib, and ic correspond to the pre-filter, post-filter, and perfectly filtered cases for a perfect Gaussian beam profile. iia, iib, and iic correspond to the same cases, but for a non-ideal telescope beam.

The results of the calibration routine are shown in Figure 10. The distribution of signal maxima appears Gaussian with a full-width-half-maximum (FWHM) of $\sim 1 \%$. The phase slope, on the other hand, appears bimodal. This is likely due to issues with the unwrapping routine, inserting parasitic factors of $\pi$ into the signal. We are investigating this further. Each mode, however, has a small FWHM as desired, indicating long-term stability of the instruments.

\section{Conclusions}

Using maps of neutral hydrogen to measure the matter power spectrum is an ambitious task. Systematics in hydrogen intensity mapping experiments related to calibration and antenna beam mapping must be understood at extreme precision in order to obtain the $21 \mathrm{~cm}$ signal through strong galactic foregrounds. Here we present two promising techniques used on the Tianlai Pathfinder Dish Array. The We will continue to develop these techniques to Tianlai Dish Array data, which will be shared with the general line intensity mapping community with the hope of application to 
future large-scale experiments. These experiments promise to make important contributions to the field of cosmology by measuring the dark energy equation of state and beyond.
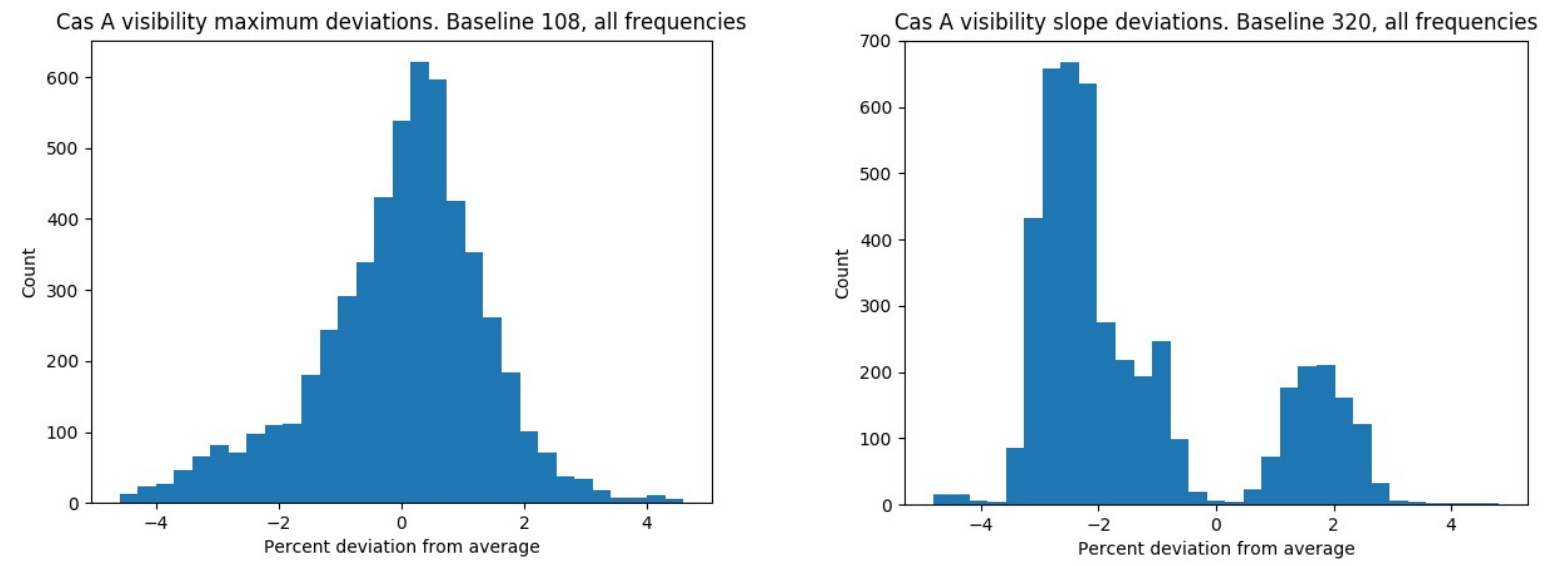

Figure 10 - Percent deviations of (left) visibility magnitude maxima and (right) visibility phase slope for a point source crossing over the Tianlai Dish Array.

\section{Acknowledgements}

Thanks to the Wisconsin Space Grant Consortium - Graduate Research Fellowship for supporting this work. Thanks to my advisor, Peter T. Timbie as well as group members Anh Phan, Santanu Das, Calvin Osinga, Gage Siebert, and Yanlin Wu, and collaborators John Marriner, Albert Stebbins, and Gregory Tucker.

\section{References}

[1] Abbott, T. M. C., et al. "Dark energy survey year 1 results: cosmological constraints from galaxy clustering and weak lensing." Physical Review D 98.4 (2018): 043526.

[2] Adam, R., et al. "Planck 2015 results-I. Overview of products and scientific results." Astronomy \& Astrophysics 594 (2016): A1

[3] Bowman, Judd D., et al. "An absorption profile centred at 78 megahertz in the sky-averaged spectrum." Nature 555.7694 (2018): 67.

[4] Chang, Tzu-Ching, et al. "An intensity map of hydrogen $21-\mathrm{cm}$ emission at redshift $\mathrm{z} \approx 0.8$." Nature 466.7305 (2010): 463.

[5] Das, Santanu, et al. "Progress in the Construction and Testing of the Tianlai Radio Interferometers." arXiv preprint arXiv:1806.04698 (2018).

[6] Hinshaw, Gary, et al. "Nine-year Wilkinson Microwave Anisotropy Probe (WMAP) observations: cosmological parameter results." The Astrophysical Journal Supplement Series 208.2 (2013): 19.

[7] Keating, Garrett K., et al. "First Results from COPSS: The CO Power Spectrum Survey." The Astrophysical Journal 814.2 (2015): 140.

[8] Kovetz, Ely D., et al. "Line-Intensity Mapping: 2017 Status Report." arXiv preprint arXiv:1709.09066 (2017). [9] L. I. G. O. Scientific. "GW170104: observation of a 50-solar-mass binary black hole coalescence at redshift 0.2." Physical Review Letters 118.22 (2017): 221101..

[10] LSST Dark Energy Science Collaboration. "Large synoptic survey telescope: dark energy science collaboration." arXiv preprint arXiv: 1211.0310 (2012).

[11] Parsons, Aaron R., and Donald C. Backer. "Calibration of low-frequency, wide-field radio interferometers using delay/delay-rate filtering." The Astronomical Journal 138.1 (2009): 219. 
[12] Riess, Adam G., et al. "Observational evidence from supernovae for an accelerating universe and a cosmological constant." The Astronomical Journal 116.3 (1998): 1009.

[13] Wu, Fengquan et. al. "The Tianlai Dish Array: design, operation and performance of a prototype transit radio interferometer." arXiv to appear (2019). 